\title{
Image Registration with Local Rigidity Constraints
}

\author{
Jan Modersitzki \\ Institute of Mathematics, University of Lübeck, Wallstraße 40, D-23560 Lübeck \\ Email: modersitzki@math.uni-luebeck.de
}

\begin{abstract}
Registration is a technique nowadays commonly used in medical imaging. A drawback of most of the current registration schemes is that all tissue is being considered as non-rigid. Therefore, rigid objects in an image, such as bony structures or surgical instruments, may be transformed non-rigidly. In this paper, we integrate the concept of local rigidity to the FLexible Image Registration Toolbox (FLIRT). The idea is to add a penalty for local non-rigidity to the cost function and thus to penalize non-rigid transformations of rigid objects. As our examples show, the new approach allows the maintenance of local rigidity in the desired fashion. For example, the new scheme is able to keep bony structures rigid during registration.
\end{abstract}

\section{Introduction}

The incorporation of pre-knowledge in registration is a key for getting meaningful results. For many registration tasks, the images inhibits an classification of soft and hard tissue. It thus seems to be natural to ask for transformations keeping hard tissue rigid. However, current registration schemes consider all parts of the tissue as non-rigid [1]. As a consequence rigid objects, such as bony structures or surgical instruments, can be transformed non-rigidly. Other consequences are that tumor growth between follow-up images may be concealed, or that structures containing contrast material in only one of the images may be compressed by the registration scheme.

Starting with the variational framework of the FLexible Image Registration Toolbox (FLIRT) [2,3], we integrate the concept of local rigidity in terms of an additional penalty term. For a transformation, rigidity is measured by linearity, orthogonality, and orientation preservation.

We also compared our approach to the non-rigidity penalized but B-spline based scheme in [1]. As it turned out, the FLIRT approach gives visually more pleasing results: a perfect match (i.e. transformed template equals reference) is achieved with a much more regular transformation; see, e.g., Figure 1.

\section{State of the art and new contribution}

There are currently two main numerical approaches to image registration. The first one is based on an expansion of the wanted transformation in terms of 
Fig. 1. Example from [1]: reference and template (first column), B-spline results taken from [1] without and with penalty (middle column), and FLIRT results without and with penalty (right column); all four transformations lead to a perfect match

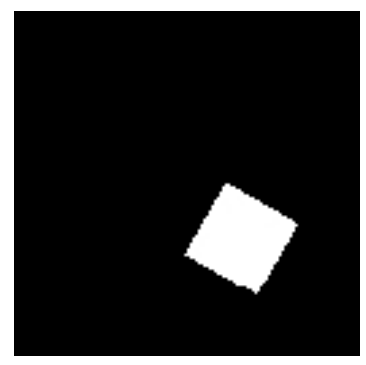

(a) reference

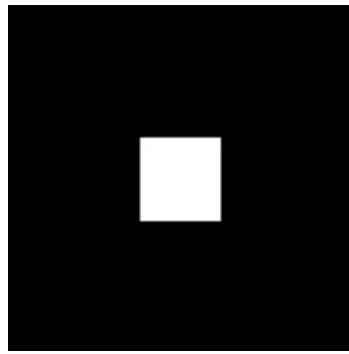

(d) template

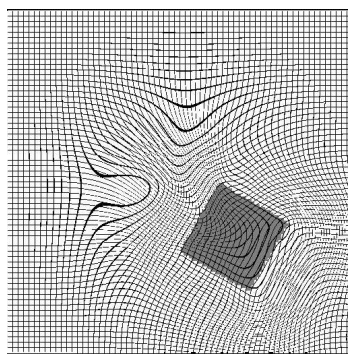

(b) no penalty

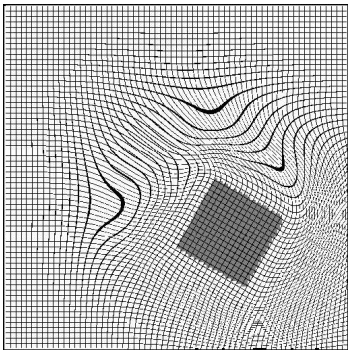

(e) penalty

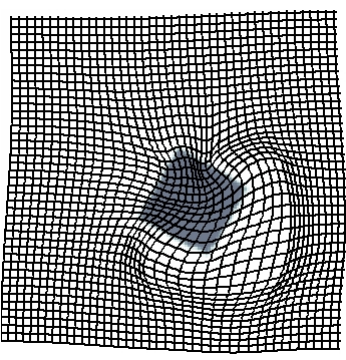

(c) no penalty

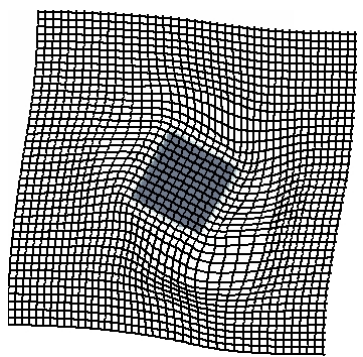

(f) penalty

B-splines [4] and the other one is based on the more general variational framework [3]. Both approaches principally allow for the integration of additional pre-knowledge in terms of a penalty, like, e.g. local rigidity. For the B-spline approach this has been implemented in [1], while the objective of this paper is the integration of a local rigidity penalty into FLIRT. In contrast to schemes with a spatially variant regularization parameter [5], where weights are given to local elasticity, the new approach explicitly penalizes non-rigidity.

The integration of application conform pre-knowledge like, e.g., local rigidity, is an important step towards improved registration. Users are much more confident to the results if important or obvious structures (like bones or surgical instruments) are transformed in a meaningful way.

\section{Methods}

We use the powerful variational framework for image registration, see $[3,6,7]$ for details. The objective is to minimize a joint functional $J$ with respect to the transformation $y$, where

$$
J(y)=D(T(y), R)+\alpha S\left(y-y^{\mathrm{kern}}\right)+\beta C(y)
$$


Here $R$ and $T$ are the reference and template image, respectively, $T(y)$ is the transformed template image, $D$ is a distance measure of choice, $S$ is a regularizer (e.g. the elastic potential), $y^{\text {kern }}$ models the kernel of the regularization, and $\alpha$ is a regularization parameter compromising between similarity and regularity. The new part is hidden in the penalty (or soft constraints) $C$, where in this paper we used local rigidity. Rigidity is measured via linearity $\left(\partial_{i, j} y_{k}=0\right)$, orthogonality $\left(\nabla y^{\top} \nabla y=I\right)$, and orientation preservation (det $\left.\nabla y=1\right)$, where $\nabla y$ denotes the Jacobian of the transformation. For a convenient implementation in a multi-level framework, the non-rigidity penalty is computed on a pixel/voxel basis and the final penalty is given as a weighted sum, where zero weights are assigned to regions which are not to be penalized; see Figure $3(\mathrm{j})$ for an example.

\section{Results}

We tested our implementation on a variety of examples. Due to page limitations, we can only present two intuitive and representative examples. Our first example is a repetition of the experiment performed in [1], see Figure 1. From these results, we see the effect of local rigidity constraints placed at non-zero locations in the moving template itself: as expected, both approaches keep the square rigid. However, a direct comparison of the two schemes is delicate. The B-spline implementation uses a backward interpolation scheme while the FLIRT implementation uses a forward scheme (in fact, for the FLIRT registration we interchanged reference and template in order to make the grids comparable). Moreover, the B-spline implementation obviously uses inappropriate vanishing Dirichlet boundary conditions (BC) (i.e. fixing the boundary of the domain), while the FLIRT approach is based on vanishing Neumann BC's. To be precise, the results of the FLIRT scheme is a global rigid transformation (which is the expected solution for this problem), but we didn't make use of the overall FLIRT capacities and do not use kernel information. Even under this artificial limitations, we find the FLIRT results superior to the B-spline results: the FLIRT transformations obtained with and without penalty are much smoother and local than the ones obtained by the B-spline approach.

A more realistic but still intuitive example is presented in Figure 2, see also [8, $3]$. Note that the template image shows a global rotation of approximately 25 degrees which outrules the B-spline approach with Dirichlet BC. In this example we make the middle finger of the hand to be rigid (see Figure $3(\mathrm{j})$ ). Figure 2 shows FLIRT results without $(\beta=0)$ as well as with penalty $(\beta=0.01)$. For both variants, we picked $\alpha=500$. As it apparent, the penalized approach does keep the finger rigid while the unconstrained does not; see particularly the plots of $\operatorname{det}(\nabla y)$ (see Figure 3(m) and 3(n)).

\section{Discussion}

The incorporation of pre-knowledge in image registration is a key for reliable results. For many registration tasks, soft and hard tissue can often be identified 
and it seems to be natural to ask for transformations keeping the hard tissue rigid.

The concept of local rigidity has been implemented in a B-splines framework by [1]. The purpose of this paper is the integration into the more general variational FLIRT framework. Our results, of which only two representative are shown in this short paper, clearly indicates that the penalized approach keeps structures like bones locally rigid and thus leads to improved registration results. For the examples presented in [1] we obtain visually more pleasing results. Compared to the alternative B-spline approach, the FLIRT approach is much more flexible. For example, it also allows the incorporation of rigidity of nearby structures, where in the B-spline approach, one has to add "enough" control points in a possible "small" gap. This can lead to very dense and/or unstructured control point grids. Moreover, the FLIRT approach allows an appropriate handling of boundary conditions which again adds to a superior overall result.

\section{References}

1. Staring M, Klein S, Pluim J. Nonrigid registration using a rigidity constraint. Procs SPIE 2006;6144:1-10.

2. Fischer B, Modersitzki J. Flirt. A flexible image registration toolbox. LNCS 2003;2717:261-270.

3. Modersitzki J. Numerical Methods for Image Registration. Oxford University Press, 2004.

4. Rueckert D, Sonoda L, Hayes C, et al. Non-rigid registration using free-form deformations. IEEE Trans Med Imaging 1999;18:712-721.

5. Kabus S, Franz A, Fischer B. Variational image registration with local properties. LNCS 2006;92-100.

6. Fischer B, Modersitzki J. Large scale problems arising from image registration. GAMM Mitteilungen 2004;27:104-120.

7. E Haber, Modersitzki J. A multilevel method for image registration. SIAM J Sci Comput 2006;27:1594-1607.

8. Amit Y. A nonlinear variational problem for image matching. SIAM J Sci Comput 1994;15:207-224. 
Fig. 2. Results for hand example: data and mask (first column), without penalty ( $\alpha=$ $500, \beta=0$; second column), with penalty $\left(\alpha=500, \beta=10^{-2}\right.$; third column), map of $\operatorname{det}(\nabla y)$, where the "blockyness" is multi-level related (last row)

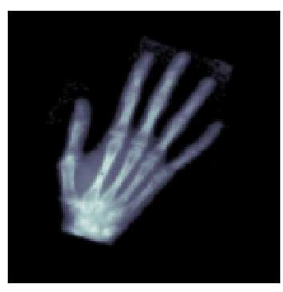

(a) template

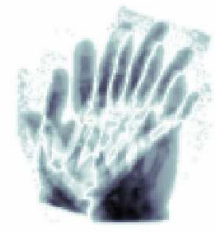

(d) difference

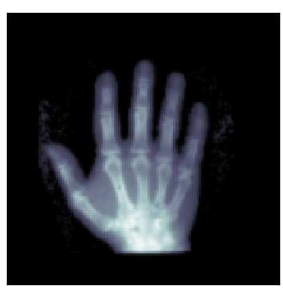

(g) reference

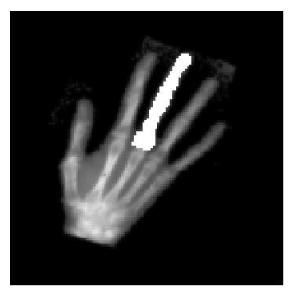

(j) weight

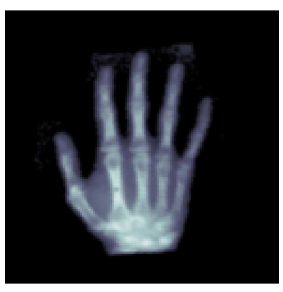

(b) $T\left(y^{\beta=0}\right)$

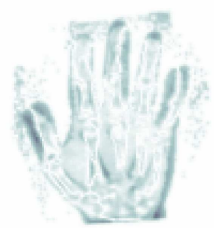

(e) $\left|T\left(y^{\beta=0}\right)-R\right|$

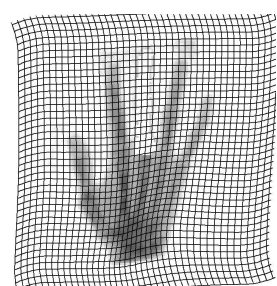

(h) $T\left(y^{\beta=0}\right)$

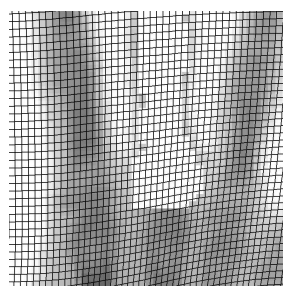

(k) ROI of grid

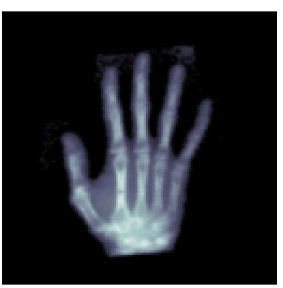

(c) $T\left(y^{\beta=0.01}\right)$

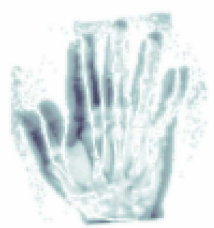

(f) $\left|T\left(y^{\beta=0.01}\right)-R\right|$

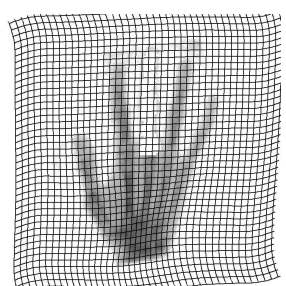

(i) $T\left(y^{\beta=0.01}\right)$

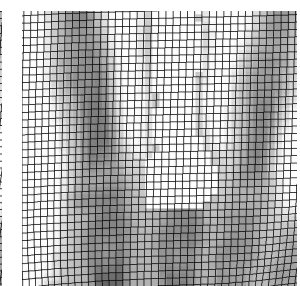

(l) ROI of grid

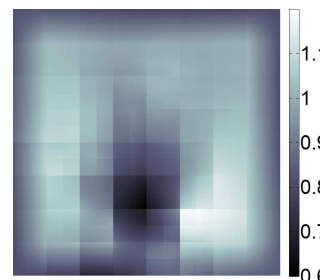

(m) $\operatorname{det}\left(\nabla y^{\beta=0}\right)$

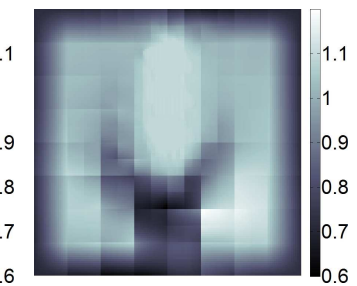

(n) $\operatorname{det}\left(\nabla y^{\beta=0.01}\right)$ 